\title{
Coordination of Neuronal Activity in Developing Visual Cortex by Gap Junction-Mediated Biochemical Communication
}

\author{
Karl Kandler and Lawrence C. Katz \\ Howard Hughes Medical Institute and Department of Neurobiology, Duke University Medical Center, Durham, North \\ Carolina 27710
}

During brain development, endogenously generated coordinated neuronal activity regulates the precision of developing synaptic circuits (Shatz and Stryker, 1988; Weliky and Katz, 1997). In the neonatal neocortex, a form of endogenous coordinated activity is present as locally restricted intercellular calcium waves that are mediated by gap junctions (Yuste et al., 1992). As in other neuronal and non-neuronal systems, these coordinated calcium fluctuations may form the basis of functional cell assemblies (for review, see Warner, 1992; Peinado et al., 1993b). In the present study, we investigated the cellular mechanisms that mediate the activation of neuronal domains and the propagation of intercellular calcium waves in slices from neonatal rat neocortex. The occurrence of neuronal domains did not depend on intercellular propagation of regenerative electrical signals because domains persisted after blockade of sodium and calcium-dependent action potentials. Neuronal domains were elicited by intracellular infusion of ino-

In the mammalian visual system, neuronal activity seems to be required for the emergence of several basic circuits, including the segregation of retinal ganglion fibers into eye-specific layers in the lateral geniculate nucleus and the development of ocular dominance columns and orientation columns in the primary visual cortex (for review, see Katz and Shatz, 1996). Because all of these structures can emerge before visual experience (Shatz, 1983; Chapman et al., 1996; Horton and Hocking, 1996), formation is likely to be driven by endogenously generated activity patterns (Shatz and Stryker, 1988; Weliky and Katz, 1997). Coordinated, endogenous activity has been described both in the immature retina in the form of traveling activity waves mediated by nicotinergic synaptic transmission (Galli and Maffei, 1988; Meister et al., 1991; Feller et al., 1996) and in the early postnatal neocortex in the form of neuronal domains (Yuste et al., 1992).

In brain slices prepared from the early postnatal rat neocortex, neuronal domains occur as spontaneous, locally restricted intercellular calcium waves that originate in one or a few centrally

Received Aug. 1, 1997; revised Oct. 15, 1997; accepted Dec. 2, 1997.

This research was supported by National Institutes of Health Grants NS32396 to L.C.K. and EY06730 to K.K. and by postdoctoral grants to K.K. from the Deutscher Akademischer Austauschdienst and the Alexander von Humboldt Stiftung. We thank Don C. Lo, Tobias Meyer, and Tom Tucker for critical comments on an earlier version of this manuscript and Darin Nelson for developing data acquisition and analysis software.

Correspondence should be addressed to Dr. Karl Kandler, Department of Neurobiology, Box 3209, Duke University Medical Center, Durham, NC 27710.

Dr. Kandler's present address: Department of Neurobiology, University of Pittsburgh School of Medicine, E 1440 Biomedical Science Tower, Pittsburgh, PA 15261

Copyright (C) 1998 Society for Neuroscience $0270-6474 / 98 / 181419-09 \$ 05.00 / 0$ sitol trisphosphate $\left(\mathrm{IP}_{3}\right)$ but not of calcium, indicating the involvement of $\mathrm{IP}_{3}$-related second-messenger systems. Pharmacological stimulation of metabotropic glutamate receptors, which are linked to the production of $\mathrm{IP}_{3}$, elicited similarly coordinated calcium increases, whereas pharmacological blockade of metabotropic glutamate receptors dramatically reduced the number of neuronal domains. Therefore, the propagating cellular signal that causes the occurrence of neuronal domains seems to be inositol trisphosphate but not calcium. Because coordination of neuronal calcium changes by gap junctions is independent of electrical signals, the function of gap junctions between neocortical neurons is probably to synchronize biochemical rather than electrical activity.

Key words: gap junction; visual cortex; inositol trisphosphate; calcium; metabotropic glutamate receptor; development; thapsigargin

located cells from which the waves propagate over a distance of 50-100 $\mu \mathrm{m}$ (Yuste et al., 1992, 1995). Propagation of these calcium waves is mediated by gap junctions and not by synaptic transmission (Yuste et al., 1995). Because neuronal domains are circular in tangential slices and often radially elongated in coronal slices, it has been proposed that neuronal domains partition the immature neocortex into columnar patches of coordinated activity. The intercellular signals underlying this form of coordinated activity, however, are obscure.

Gap junctions allow the passage of electrical currents and small molecules up to $\sim 1 \mathrm{kDa}$ between coupled cells (for review, see, e.g., Bennett et al., 1991). In the developing neocortex, electrical coupling between neurons has been demonstrated in the ventricular zone (Lo Turco and Kriegstein, 1991) and in the cortical plate (Connors et al., 1983). Coupling is also most likely responsible for the intercellular propagation of calcium spikes in the presence of potassium channel blockers (Yuste et al., 1995) and for mediating the junctional spread of membrane depolarizations in cultured cortical neurons (Charles et al., 1996; Peinado et al., 1993b).

Despite these examples, electrical coupling between neurons in the neocortex seems to be too weak to be responsible for the synchronization of neuronal activity during the occurrence of a neuronal domain (Connors et al., 1983; Peinado et al., 1993b). An alternative possibility is that gap junctions synchronize neuronal activity by coordinating biochemical activity rather than electrical activity. In non-neuronal cells, gap junctions mediate intercellular biochemical communication via the direct exchange of secondmessenger molecules (Boitano et al., 1992; Allbritton and Meyer, 1993; for review, see Sanderson, 1995), thus raising the possibility 
that neuronal activity might be coordinated by biochemical communication through gap junctions.

In the present study, we elucidated the cellular mechanisms by which gap junctions coordinate neuronal behavior in the developing rat neocortex by using calcium-imaging techniques in brain slices. We found that neuronal domains are initiated by stimulation of metabotropic glutamate receptors and by intracellular increase in the second-messenger molecule inositol trisphosphate $\left(\mathrm{IP}_{3}\right)$ that releases calcium from intracellular stores. In addition to its role as an intracellular messenger, $\mathrm{IP}_{3}$ also seems to be the intercellular signal molecule that diffuses between coupled cells and therefore underlies the propagation of neuronal calcium waves. This cascade of events strongly supports the hypothesis that neuronal gap junctions in the developing neocortex coordinate the biochemical activity among coupled cell assemblies.

Parts of this paper have been published previously in abstract form (Kandler and Katz, 1995).

\section{MATERIALS AND METHODS}

Slices (300-400 $\mu \mathrm{m}$ thick) were prepared from the visual cortex of postnatal day 0 (P0)-P8 rats and were stained with fura-2 AM (Molecular Probes, Eugene, OR) as described previously (Yuste et al., 1995) with the only difference that $\mathrm{Mg}^{2+}$-containing artificial CSF (ACSF) (composition in mM: $\mathrm{NaCl} 124, \mathrm{MgSO}_{4} 1.3, \mathrm{CaCl}_{2} 3.1, \mathrm{KCl} 5, \mathrm{KH}_{2} \mathrm{PO}_{4} 1.25$, glucose 10 , and $\mathrm{NaHCO}_{3} 26$, pH 7.4, when bubbled with $95 \% \mathrm{O}_{2} / 5 \%$ $\mathrm{CO}_{2}$ ) was used during both staining and imaging. Calcium imaging was performed at room temperature $\left(21-25^{\circ} \mathrm{C}\right)$ using an upright microscope (Axioskope; Zeiss) equipped with an intensified charge-coupled device camera (Hamamatsu) coupled to an image processor (Imaging Technologies Series 151). Single excitation images $(385 \mathrm{~nm})$ were acquired every $1-10 \mathrm{sec}$, and after background subtraction, the average of 16 frames was stored on an optical disk recorder (Panasonic TQ 2028F) for off-line analysis. Individual frames were digitized and further processed using the program National Institutes of Health Image (ftp://zippy.nimh.gov/ $\mathrm{pub} /$ nih-image). To minimize phototoxicity caused by prolonged illumination, we elicited neuronal domains by transiently decreasing the temperature of the superfusing ACSF by $3-5^{\circ} \mathrm{C}$, as described previously (Yuste et al., 1995). For domain detection, a reference image before a temperature drop (TD) was subtracted from subsequent images. Subtracted images were then smoothed by convolution with a $7 \times 7$ Gaussian kernel, and intensity changes were expressed as $\Delta F / F$ (in percent). A neuronal domain was defined as a decrease in the fura- 2 signal in more than five neighboring neurons occurring in the same frame. The size of domains was measured by applying a threshold of twice the SD of the pixel values in the image (Yuste et al., 1995). All values are expressed as the arithmetic mean \pm SEM.

Conventional whole-cell patch-clamp recording techniques (Blanton et al., 1989) were used to form gigaohm seals on neurons under visual control (Axioskope equipped with $63 \times$ and $40 \times$ objectives and Nomarski optics; Zeiss). Patch pipettes (4-9 $\mathrm{M} \Omega$ ) were filled with internal solution (composition in mM: cesium gluconate 110 or $\mathrm{KCl} 130, \mathrm{MgCl}_{2} 1$, $\mathrm{CaCl}_{2}$ 1, EGTA 11, and HEPES 10, pH 7.2; in some experiments the solution also contained $50-100 \mu \mathrm{M}$ fura-2 pentapotassium). For intracellular $\mathrm{IP}_{3}$ infusions, $1 \mathrm{~mm}$ inositol 1,4,5-trisphosphate or $50-100 \mu \mathrm{M}$ inositol 2,4,5-trisphosphate, a nonhydrolyzable analog, was added to the internal solution. Relatively high $\mathrm{IP}_{3}$ concentrations were used to ensure the rapid intracellular inf usion of a sufficient amount of $\mathrm{IP}_{3}$. For calcium injections, the internal solution contained no EGTA and contained $1 \mathrm{~mm}$ $\mathrm{CaCl}_{2}$ and in some cases $200 \mu \mathrm{M}$ fluo-3 pentapotassium. In some cases, intracellular calcium was increased by the activation of voltage-gated calcium channels by a train $(10-40 \mathrm{sec})$ of depolarizing voltage steps $(50$ msec in duration; $10 \mathrm{~Hz}$ ). These depolarizations consistently elicited regenerative action potentials that, because of the presence of $2-5 \mu \mathrm{M}$ TTX, were interpreted as calcium action potentials. After the formation of gigaohm seals, image capturing was started and followed by rupture of the cell membrane.

To deplete intracellular calcium stores with thapsigargin, we dissolved fura-2 AM in DMSO that also contained $1 \mathrm{~mm}$ thapsigargin (Sigma, St. Louis, MO), resulting in a final concentration of $10 \mu \mathrm{M}$ thapsigargin in the fura-2 AM-staining solution. Unless otherwise noted, all chemicals were purchased from Sigma.

\section{RESULTS}

In the following sections, we first describe experiments that demonstrate that neuronal calcium waves result from the release of calcium from intracellular stores, suggesting that neuronal domains are generated by the intercellular propagation of biochemical rather than electrical signals. We next show that the initiation of neuronal domains involves stimulation of metabotropic glutamate receptors, which are linked to the production of intracellular $\mathrm{IP}_{3}$. Finally, we demonstrate that $\mathrm{IP}_{3}$, not calcium ions, acts as the propagating signal between coupled cells.

Changes in the intracellular calcium concentration were monitored in brain slices from the occipital cortex of early postnatal rats stained with the calcium indicator fura-2 AM. All experiments were conducted in the presence of the sodium channel antagonist tetrodotoxin $(2-5 \mu \mathrm{M})$ to block sodium-dependent action potentials and synaptic transmission. Because spontaneous neuronal domains occur sporadically at long intervals of $\sim 4 \mathrm{~min}$, the acquisition of a sufficient number of domains for quantification would have required continuous imaging over several hours. To minimize photobleaching and phototoxicity associated with prolonged illumination periods, we used small transient temperature drops $\left(3-5^{\circ} \mathrm{C}\right)$ to elicit neuronal domains. Previous studies have demonstrated that TD-elicited neuronal domains are indistinguishable from spontaneously occurring neuronal domains (Yuste et al., 1995). Consistent with these studies, TD consistently triggered the appearance of neuronal domains (average, $4.5 \pm 1.1$ domains per TD; $n=17$ slices) that were randomly located throughout the cortical depth and that covered an average area of $2782 \pm 193 \mu \mathrm{m}^{2}(n=79$ domains) (Figs. $1 A, 2)$.

\section{Activation of neuronal domains depends on the release of calcium from intracellular stores}

To address the cellular mechanisms that underlie the activation of neuronal domains, we first determined the sources of calcium responsible for the increase in free intracellular calcium concentration $\left(\left[\mathrm{Ca}^{2+}\right]_{\mathrm{i}}\right)$. The occurrence of a neuronal domain begins in one or a few "trigger cells" from which a calcium wave propagates radially (Yuste et al., 1995). Because gap junctions are permeable to electrical current as well as to small second-messenger molecules (Bennett and Goodenough, 1978; Saez et al., 1989; Bennett et al., 1991), intercellular calcium waves could be generated either by the intercellular propagation of electrical signals, such as calcium spikes (Yuste et al., 1995), or by the intercellular diffusion of chemical signals, such as calcium ions or small secondmessenger molecules (Saez et al., 1989; Boitano et al., 1992; for review, see Sanderson, 1995). To distinguish between these two possibilities, we applied TDs in the presence of 2-5 mM nickel chloride, which, at these concentrations, blocks both low voltageand high voltage-activated calcium channels ( $\mathrm{Gu}$ et al., 1994). Although nickel completely blocked depolarization-induced calcium spikes ( $0.5 \mathrm{~mm} \mathrm{NiCl}$ and $2 \mu \mathrm{M}$ TTX; $n=5$ neurons; data not shown), TDs in the presence of nickel still consistently elicited neuronal domains with an average frequency and area indistinguishable from those of controls (frequency, $5.2 \pm 1.0$ domains/ TD; $n=14$ slices; $p>0.1$, Student's $t$ test; area, $3190 \pm 279 \mu \mathrm{m}^{2}$; $n=72$ domains; $p>0.1$, Student's $t$ test; Figs. $1 B, 2)$.

These findings indicate that the junctional propagation of fast electrical signals such as calcium action potentials and that calcium entry via voltage-gated calcium channels are not required for the activation of neuronal domains. We next tested whether neuronal domains are elicited by the release of calcium from internal stores by depleting intracellular calcium stores with thap- 


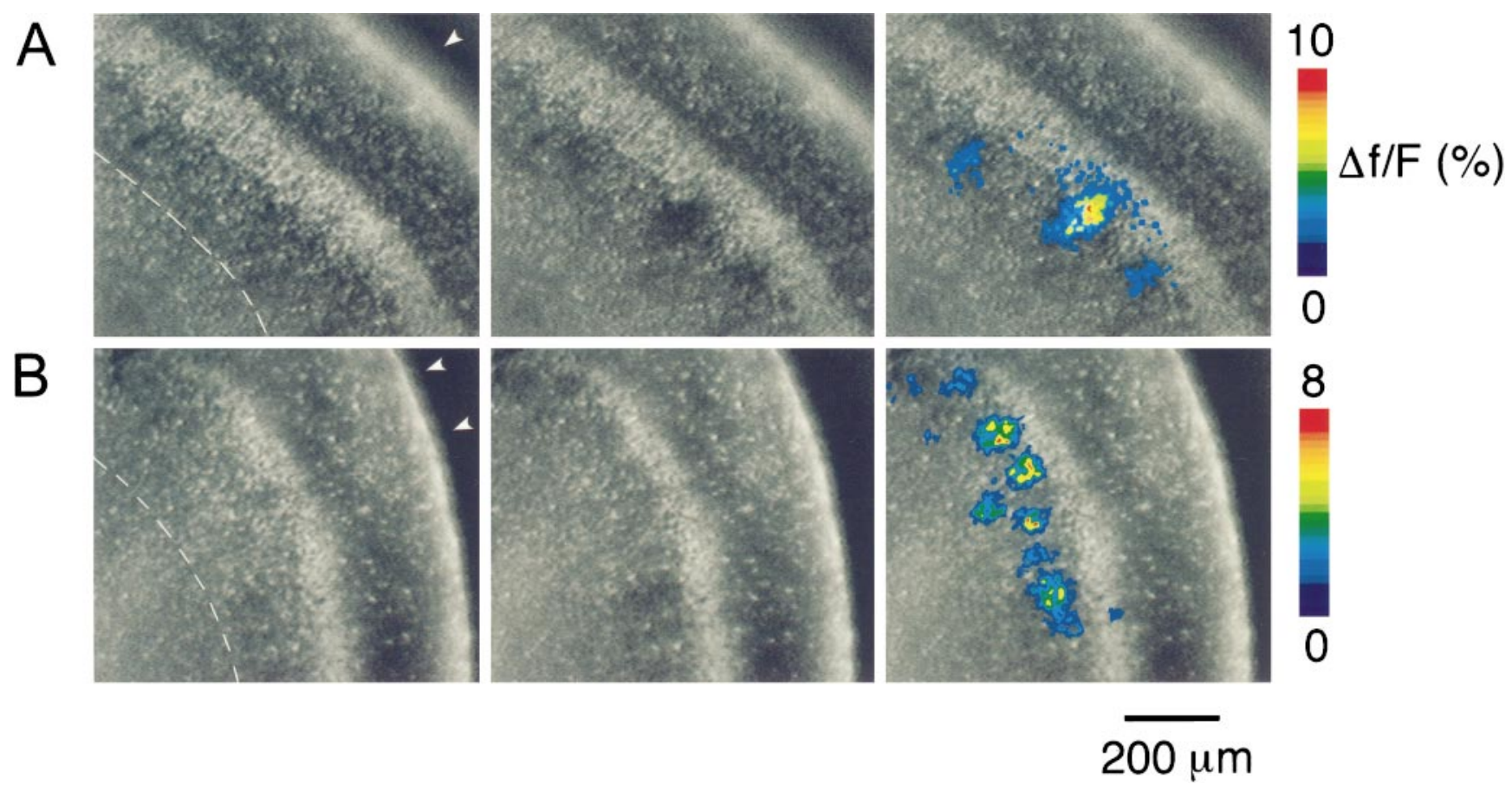

Figure 1. Neuronal domains in fura-2-stained slices in neonatal rat visual cortex. Slices were illuminated with $385 \mathrm{~nm}$ light at which fura-2 emission decreases with increasing calcium concentration. Video images were taken before (left) and during (middle) the occurrence of neuronal domains. On the right, the changes in the fura-2 fluorescence are expressed in pseudocolor as $\Delta F / F$ (in percent) and overlaid onto the images shown in the middle. Each individual image is the average of 16 background-subtracted single frames taken at video rate. The pial surface is indicated by the arrowheads; the white matter is identified by the dashed line. A, Neuronal domains elicited by temperature drop under control conditions $(2 \mu \mathrm{M}$ TTX). Coronal slice of a P3 rat. $B$, Neuronal domains elicited by temperature drop in the presence of $2 \mathrm{mM} \mathrm{Ni}^{2+}$ and $2 \mu \mathrm{M}$ TTX. These neuronal domains are similar in size and shape to those observed under control conditions, indicating that neither sodium-dependent action potentials nor extracellular $\mathrm{Ca}^{2+} \mathrm{entry}^{2}$ is required for the occurrence of neuronal domains. Coronal slice of a $\mathrm{P} 2$ rat.

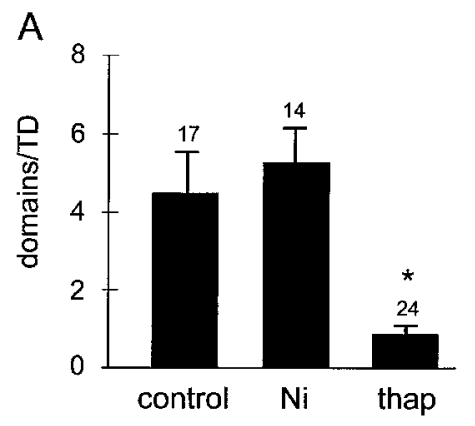

B

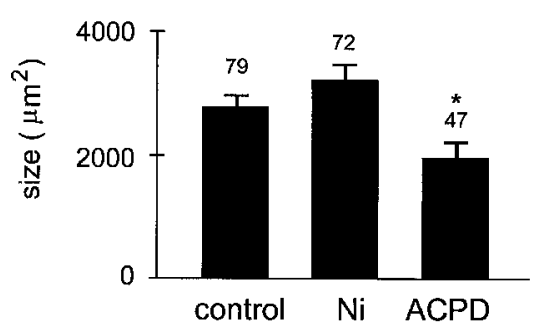

Figure 2. Effects of nickel, thapsigargin, and t-ACPD on the number and size of neuronal domains. $A$, Average number of neuronal domains elicited by temperature drop under control conditions and in the presence of $2 \mathrm{~mm}$ nickel chloride $(\mathrm{Ni})$ or $10 \mu \mathrm{M}$ thapsigargin (thap). Blockade of voltage-gated calcium channels with $2 \mathrm{~mm}$ nickel had no effect on the number of domains. In contrast, depletion of intracellular calcium stores with $10 \mu \mathrm{M}$ thapsigargin almost completely abolished neuronal domains $(p<0.01$, Student's $t$ test). $B$, Average size of neuronal domains elicited by temperature drop under control conditions and in the presence of $2 \mathrm{~mm}$ nickel $(\mathrm{Ni})$ and elicited by bath application of t-ACPD $(40-100 \mu \mathrm{m})$. Neuronal domains elicited by t-ACPD were smaller $(p<0.01)$ than were domains elicited by temperature drop. In all cases, the bath solution contained $2 \mu \mathrm{M}$ TTX. Numbers above bars indicate the number of slices $(A)$ or number of neuronal domains $(B)$. Asterisks indicate a significant difference ( $p \leq 0.05$; student's $t$ test). sigargin $(10 \mu \mathrm{M})$, an endoplasmic reticular $\mathrm{Ca}^{2+}$-ATPase inhibitor. In thapsigargin-treated slices, the number of neuronal domains decreased dramatically from $4.5 \pm 1.1$ domains/TD $(n=17$ slices) to $0.8 \pm 1.1$ domains/TD $(n=24$ slices; $p<0.01$; Fig. $2 A)$. Thapsigargin had no effect on calcium increases in single cells resulting from $\mathrm{KCl}$-induced depolarizations $(60 \mathrm{~mm})(n=8$ slices; data not shown). Taken together, these results demonstrate that the activation of cortical neuronal domains requires calcium release from intracellular stores.

\section{Activation of metabotropic glutamate receptors elicits neuronal domains}

Because the activation of neuronal domains results from calcium release from intracellular stores, we next investigated whether neuronal domains could be elicited by neurotransmitters that activate internal calcium stores. Because the metabotropic glutamate receptors mGluR1 and mGluR5 are abundant in the immature neocortex and are linked to the production of $\mathrm{IP}_{3}$ (Dudek et al., 1989; Fotuhi et al., 1993; Bevilacqua et al., 1995) and to the release of calcium from intracellular stores (for review, see Berridge, 1993), we tested whether stimulation of mGluR elicits neuronal domains. Bath application of the agonist $(1 S, 3 R)-1$ aminocyclopentane-1,3-dicarboxylic acid (t-ACPD; 40-100 $\mu \mathrm{M}$ ), which increases $\mathrm{IP}_{3}$ in cortical neurons (Challiss et al., 1994), triggered numerous neuronal domains throughout the cortical depth $(5.2 \pm 2.7$ domains/treatment; $n=9$ slices) (in Fig. $3 A$, several domains in upper layers are shown). Although the overall 

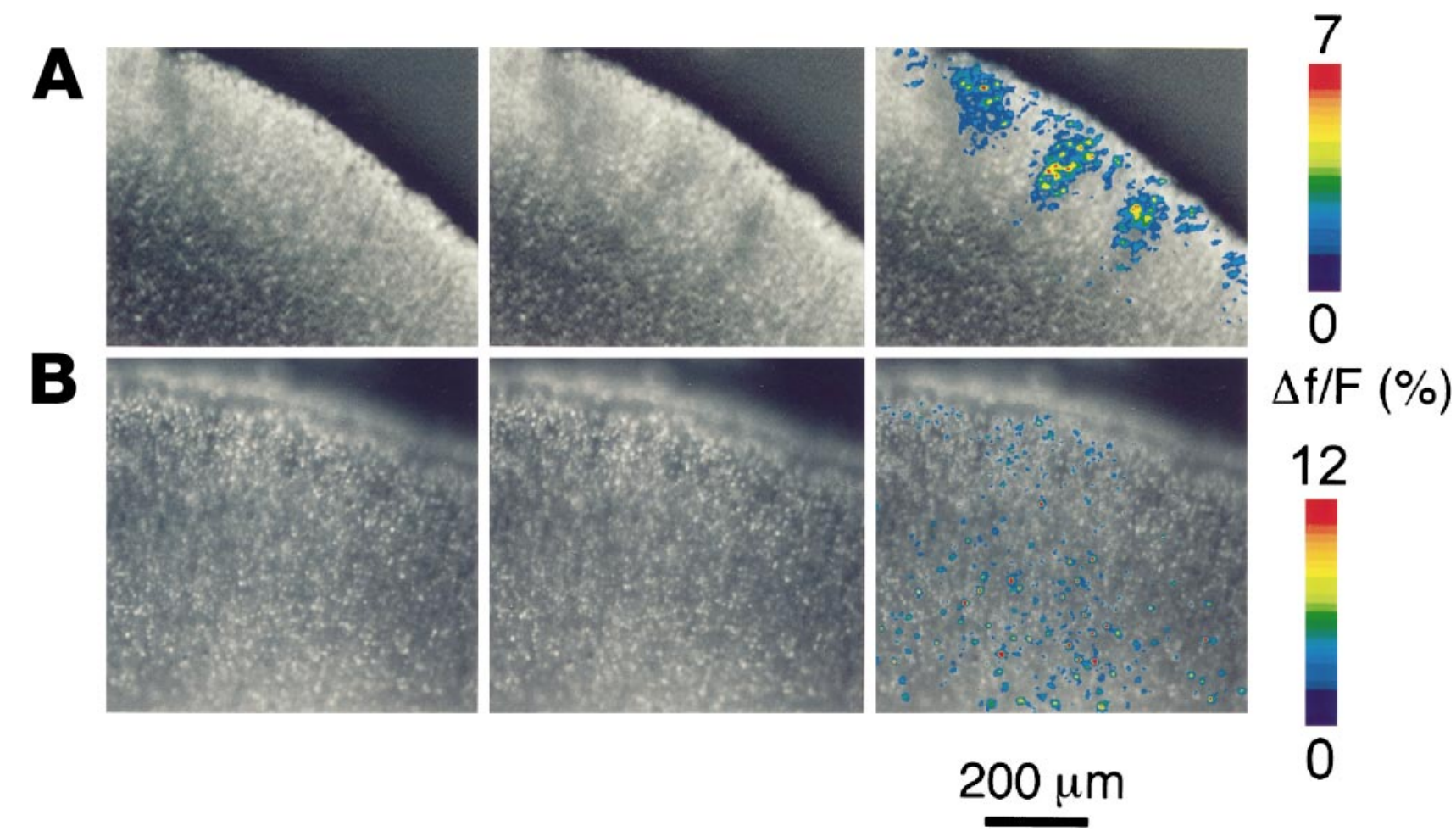

Figure 3. A, Activation of the metabotropic glutamate agonist t-ACPD elicits neuronal domains. The left and middle images were taken before and during the application of $40 \mu \mathrm{M}$ t-ACPD. In the right image, changes in fura-2 emission are pseudocolor coded as $\Delta F / F$ (in percent) and overlaid onto the middle image. Each individual image is the average of 16 background-subtracted frames. Numerous neuronal domains are visible, as are individual cells, the $\left[\mathrm{Ca}^{2+}\right]_{\mathrm{i}}$ of which increased by activation of metabotropic glutamate receptors. $B$, t-ACPD-elicited neuronal domains depend on functional gap junctions. In the presence of the gap junction blocker octanol (1 mM), application of $200 \mu \mathrm{M} \mathrm{t}$-ACPD elicits only single-cell responses but no neuronal domains. The pial surface is to the upper right in $A$ and $u p$ in $B$. A, Coronal slice of a $\mathrm{P} 3$ rat. $B$, Coronal slice of a $\mathrm{P} 4$ rat.

shape of t-ACPD-elicited domains resembled spontaneous (Yuste et al., 1995) and TD-elicited domains (Fig. 1), they were somewhat smaller than were those elicited by TD (average area, $1963 \mu \mathrm{m}^{2} \pm 198 ; n=47$ domains) (Fig. $2 B$ ), a finding for which we currently cannot offer a plausible explanation.

In addition to neuronal domains, t-ACPD also elicited isolated calcium responses in individual neurons (Fig. $3 A$ ), perhaps because of subthreshold $\mathrm{IP}_{3}$ concentrations in these neurons or because of the existence of a subpopulation of (highly coupled) neurons capable of eliciting neuronal domains.

Because of temporal limitations of our imaging system (maximum frame rate, $1 \mathrm{~Hz}$ ), we could not exclude the possibility that t-ACPD-elicited domains resulted from direct, simultaneous activation of a group of neighboring neurons rather than from "triggering" of a few cells. We attempted to address this possibility by injecting t-ACPD locally, but because of the dimensions of neuronal domains (diameters, $\sim 50-100 \mu \mathrm{m}$ ), it was impossible to distinguish between seemingly coordinated calcium increases resulting from extracellular diffusion of injected t-ACPD and genuine neuronal domains resulting from intercellular calcium waves. Therefore, we applied t-ACPD (100-200 $\mu \mathrm{M})$ in the presence of the gap junction blocker octanol. In seven slices, bath application of t-ACPD in the presence of $1 \mathrm{~mm}$ octanol elicited calcium changes only in individual cells and never triggered neuronal domains (Fig. 3B). This indicates that t-ACPD-elicited domains were caused by stimulation of individual cells that acted as trigger cells to elicit gap junction-mediated neuronal domains. The application of the inactive enantiomer cis-ACPD (100-500 $\mu \mathrm{M})$ did not trigger neuronal domains, nor did it increase the cytosolic calcium concentration in individual neurons $(n=5$ slices; data not shown). All the effects of t-ACPD were blocked by the metabotropic glutamate receptor antagonist (+)- $\alpha$-methyl-4carboxyphenylglycine $[(+)-\mathrm{MCPG} ; 1 \mathrm{~mm} ; n=3$ slices; data not shown].

To test whether the occurrence of neuronal domains depends on the activation of mGluRs, we applied temperature drops while blocking mGluRs. Bath application of the mGluR antagonist (+)-MCPG (1 mM) substantially reduced the number of neuronal domains (MCPG, $1.3 \pm 0.5$ domains/TD; $n=21$ slices; control, $5.4 \pm 1.1$ domains/TD; $n=7$ slices; $p<0.01$; Fig. $4 A$ ), indicating that $\mathrm{mGluR}$ activation is critically involved in the initiation of neuronal domains. If $\mathrm{mGluR}$ activation is also involved in the propagation of domains, one would expect that (+)-MCPG also decreases the size of domains. However, neuronal domains that persisted in the presence of $(+)-\mathrm{MCPG}$ were similar in size to controls (MCPG, $3756 \pm 390 \mu \mathrm{m}^{2} ; n=29$ domains; age matched control, $3851 \pm 246 \mu \mathrm{m}^{2} ; n=53$ domains; $p>0.1$; Fig. $4 B$ ), suggesting that mGluR activation is responsible for the initiation of domains but not for the continued propagation of interneuronal calcium waves.

\section{Increases in intracellular inositol trisphosphate, but not calcium, trigger neuronal domains}

To test the participation of $\mathrm{IP}_{3}$ in neuronal domains directly, we infused $\mathrm{IP}_{3}$ through patch pipettes into individual cells. These $\mathrm{IP}_{3}$ infusions produced calcium waves 2-30 sec after the rupture of the cell membrane and influx of $\mathrm{IP}_{3}$ (Fig. 5). These calcium waves always originated from the injected cell and covered an 


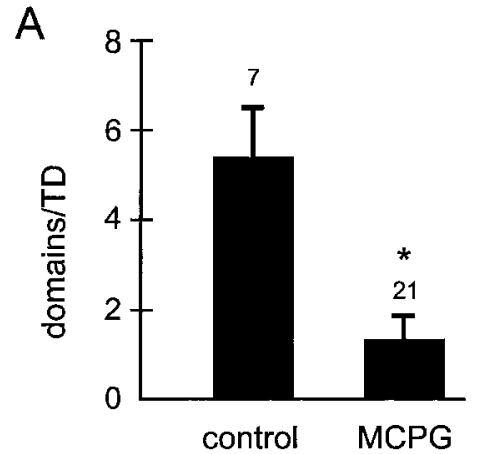

Figure 4. Metabotropic glutamate receptors are required for initiation of neuronal domains. $A$, Bath application of 1 $\mathrm{mm}(+)$-MCPG dramatically reduced the number of neuronal domains ( $p<0.01$, Student's $t$ test). $B$, Bath application of $1 \mathrm{~mm}(+)$-MCPG does not affect the size of the neuronal domains that persist, indicating that metabotropic glutamate receptors are only involved in the initiation of neuronal domains. Numbers above bars indicate the number of slices $(A)$ or number of neuronal domains $(B)$. An asterisk indicates a significant difference ( $p \leq 0.01$; student's $t$-test).
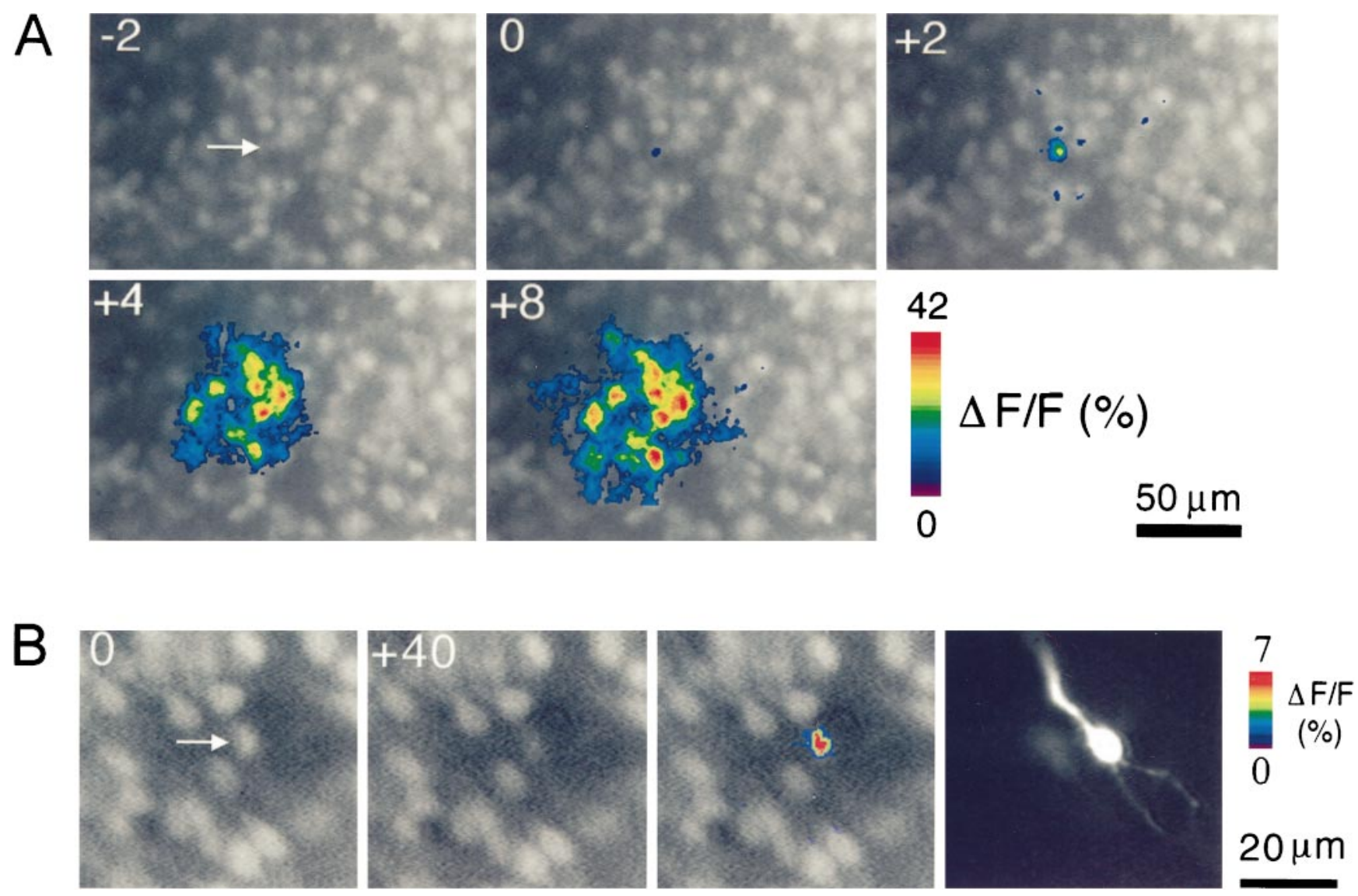

Figure 5. Neuronal domains are elicited by increasing $\left[\mathrm{IP}_{3}\right]_{\mathrm{i}}$ but not $\left[\mathrm{Ca}^{2+}\right]_{\mathrm{i}}$. A, Neuronal domains elicited by intracellular infusion of inositol $1,4,5$-trisphosphate are shown. Changes in the fura-2 fluorescence signal are pseudocolor coded as $\Delta F / F$ (in percent) and superimposed on background-subtracted video frames. The arrow points to the filled cell in the first frame that was taken 2 sec $(-2)$ before rupture of the cell membrane ( frame 0). Increasing $\left[\mathrm{IP}_{3}\right]_{\mathrm{i}}$ triggered a concentric calcium wave with a diameter of $\sim 80 \mu \mathrm{m}$. Tangential slice of a P1 rat. $B$, Increasing $\left[\mathrm{Ca}{ }^{2+}\right]_{\mathrm{i}}$ in a single cell (arrow) by depolarizing voltage steps failed to elicit intercellular calcium waves. The cell was depolarized from a holding potential of $-70 \mathrm{mV}$ to $+10 \mathrm{mV}$ by a $40 \mathrm{sec}$ train consisting of 50 -msec-long depolarizations delivered at $10 \mathrm{~Hz}$. The neuron was also filled with $100 \mu \mathrm{M}$ fluo- 3 to visualize its basic structure (right). Coronal slice of a $\mathrm{P} 3$ rat.

average area of $2164 \pm 353 \mu \mathrm{m}^{2}$ ( $n=18$ domains) that closely resembled the size of neuronal domains elicited by TD (2782 \pm $193 \mu \mathrm{m}^{2} ; n=79$ domains; $p>0.1$, Student's $t$ test). Patching neurons without $\mathrm{IP}_{3}$ in the pipette solution never triggered neuronal domains. These experiments demonstrate that the stimulation of an individual cell can trigger a neuronal domain and that $\mathrm{IP}_{3}$ alone is sufficient to trigger neuronal domains.

Previous studies in non-neuronal cells have shown that intercellular calcium waves can be mediated by the diffusion of the second messengers $\mathrm{IP}_{3}$ or calcium ions (Saez et al., 1989; Christ et al., 1992; for review, see Sanderson, 1995). To investigate the nature of the propagating molecule that causes the occurrence of cortical neuronal domains, we tested whether neuronal domains could be elicited by increasing intracellular $\left[\mathrm{Ca}^{2+}\right]_{i}$ without directly changing $\left[\mathrm{IP}_{3}\right]_{i} \cdot\left[\mathrm{Ca}^{2+}\right]_{i}$ was increased by intracellular infusion of calcium through a patch pipette that contained $1 \mathrm{~mm}$ $\mathrm{Ca}^{2+}(n=14$ cells $)$ or by activating voltage-gated calcium channels (Giffin et al., 1991) with depolarizing electrical current injections (+60 mV; $100 \mathrm{msec} ; 5 \mathrm{~Hz} ; 5-10 \mathrm{sec} ; n=12$ neurons). Both approaches consistently increased calcium levels in single neurons, but changes in $\left[\mathrm{Ca}^{2+}\right]_{\mathrm{i}}$ were always restricted to the patched cell and never triggered propagating intercellular waves 
(Fig. $5 B$ ). Thus an increase in $\left[\mathrm{IP}_{3}\right]_{\mathrm{i}}$ is required both for eliciting and propagating neuronal calcium waves.

As calcium-induced calcium release (CICR) is a prominent calcium release mechanism in cerebellar Purkinje neurons (Kano et al., 1995) and a component of calcium wave propagation between retinal Mueller cells (Keirstead and Miller, 1995), we assessed its contribution to intracellular calcium waves in cortical neurons. Blockage of CICR with ryanodine $(100 \mu \mathrm{M})$ had no significant effect on the size (ryanodine, $2330 \pm 203 \mu \mathrm{m}^{2} ; n=38$; control, $\left.2782 \pm 193 \mu \mathrm{m}^{2} ; n=79 ; p>0.1\right)$ or the frequency (ryanodine, $6.33 \pm 1.0$ domains/TD; control, $4.5 \pm 1.1$ domains/ TD; $n=17 ; p>0.1$ ) of neuronal domains. This indicates that CICR is not required for the generation of neuronal domains.

\section{DISCUSSION}

In the present study, we investigated the cellular mechanisms that generate coordinated neuronal activity in the form of gap junction-mediated intercellular calcium waves (neuronal domains) in the early developing neocortex. Our data suggest that initiation of neuronal domains consists of stimulation of metabotropic glutamate receptors, an increase in $\left[\mathrm{IP}_{3}\right]_{\mathrm{I}}$, and release of calcium from intracellular stores. Propagation of neuronal activity involves the intercellular diffusion and probably the partial regeneration of $\mathrm{IP}_{3}$. In contrast to other forms of early coordinated activity, such as retinal waves (Meister et al., 1991; Feller et al., 1996), neuronal domains are coordinated by biochemical rather than electrical activity.

\section{Neuronal domains are generated by the intercellular diffusion of inositol trisphosphate and the release of calcium from internal stores}

The results of this study indicate that neuronal domains in the developing neocortex are caused by propagation of the intercellular diffusion of the second messenger $\mathrm{IP}_{3}$ rather than by the intercellular spread of electrical signals or of calcium ions. This is supported by the following observations. First, neuronal domains persist when sodium- and calcium-dependent action potentials are blocked with TTX (Fig. $1 A$; Yuste et al., 1995) and nickel (Fig. $1 B$ ), arguing against propagation of regenerative electrical signals as the responsible mechanism. Second, neuronal domains are not elicited by single-cell depolarizations (Fig. 5B) and are not affected by blocking voltage-gated calcium channels with high concentrations of nickel, indicating that neuronal domains are not generated by passive electrotonic spread of depolarizations and subsequent activation of voltage-gated calcium channels. Third, neuronal domains are abolished after depletion of intracellular calcium stores with thapsigargin, indicating that neuronal domains depend on the release of calcium from internal stores. Fourth, neuronal domains are elicited by increasing the intracellular $\mathrm{IP}_{3}$ concentration, either by stimulation of metabotropic glutamate receptors (Fig. $3 A$ ) or by intracellular $\mathrm{IP}_{3}$ infusions (Fig. $5 A$ ), indicating that $\mathrm{IP}_{3}$ is sufficient for triggering neuronal domains. Finally, neuronal domains are not elicited by increases in the intracellular calcium concentration produced by either direct infusion of calcium or by activation of voltage-gated calcium channels (Fig. 5B), indicating that calcium is neither the intracellular trigger nor the signal that diffuses through gap junctions.

Intercellular second-messenger waves have been described previously in a variety of non-neuronal systems (for review, see, e.g., Katz, 1995) and between leech neurons (Wolszon et al., 1994). In these systems, the propagating second-messenger molecule has been characterized as either $\mathrm{IP}_{3}$ (for review, see Sanderson, 1995) or calcium (Wolszon et al., 1994). The results of the present study suggest that $\mathrm{IP}_{3}$, or perhaps one of its metabolites (for review, see Berridge, 1993), acts as the intercellular propagating molecule that causes a neuronal domain. According to our results, calcium acts as the responding molecule downstream from $\mathrm{IP}_{3}$ because increasing intracellular $\mathrm{IP}_{3}$, but not calcium alone, was sufficient to trigger neuronal domains (Fig. 5). Although one could argue that the failure of calcium infusions to elicit neuronal domains might be the result of calcium-induced uncoupling (Baux et al., 1978; Peracchia, 1978; Rao et al., 1987), such uncoupling does not occur with calcium levels as they are achieved by calcium spikes (Yuste et al., 1995) or by prolonged depolarizations, such as those routinely used for biocytin injections to visualize dye coupling in these same cells (Peinado et al., 1993a). Because single-cell depolarization also failed to trigger neuronal domains (Fig. 5B), calcium ions alone can neither trigger nor propagate cortical neuronal calcium waves. Possible reasons for this could include permeation selectivity of cortical neuronal gap junctions (for review, see Veenstra, 1996) or differences in the diffusion properties of $\mathrm{IP}_{3}$ and $\mathrm{Ca}^{2+}$ (for review, see Kasai and Peterson, 1994).

\section{Neuronal domains are initiated by activation of metabotropic glutamate receptors}

Since the discovery of spontaneous neuronal domains, the physiological signals responsible for their initiation have remained obscure. The results from the present study strongly suggest that the major physiological trigger for neuronal domains is glutamate acting via metabotropic glutamate receptors. Stimulation of mGluRs with the agonist t-ACPD elicited neuronal domains (Fig. $3 A$ ), whereas blockade of mGluRs by the antagonist (+)-MCPG dramatically reduced the number of neuronal domains (Fig. 4). The fact that (+)-MCPG did not completely prevent the occurrence of all neuronal domains (Fig. $4 A$ ) may be attributed to an incomplete blockade of all mGluRs or to the existence of additional trigger mechanisms such as other neurotransmitter receptors capable of increasing intracellular $\mathrm{IP}_{3}$ concentrations (Kendall and Nahorski, 1987; Simpson et al., 1995). A likely candidate for mediating glutamate-elicited neuronal domains is the metabotropic glutamate receptor mGluR5, which is highly expressed in early postnatal cortex and is coupled to the production of $\mathrm{IP}_{3}$ (Abe et al., 1992; Catania et al., 1994). These receptors could then be activated either by ambient glutamate or by glutamate spontaneously released from immature synapses (Lo Turco et al., 1994; Kim et al., 1995).

Although neuronal domains can be readily observed in slices, whether they also occur in vivo has been questioned, because a physiologically plausible triggering mechanism was absent. Our finding that glutamate, the major excitatory cortical neurotransmitter, can initiate neuronal domains in slices, however, makes the presence of neuronal domains in vivo more plausible.

\section{Proposed model for the initiation and propagation of cortical calcium waves}

Based on our results, we propose the following model for the initiation and propagation of cortical calcium waves (Fig. 6). Neuronal domains are initiated by glutamate, probably released from developing synapses, which acts on metabotropic glutamate receptors. These in turn are linked to G-proteins that activate phospholipase C. Stimulation of this cascade results in production of $\mathrm{IP}_{3}$ and the subsequent release of calcium from intracellular $\mathrm{IP}_{3}$-sensitive stores. In addition to its role of mediating this direct 


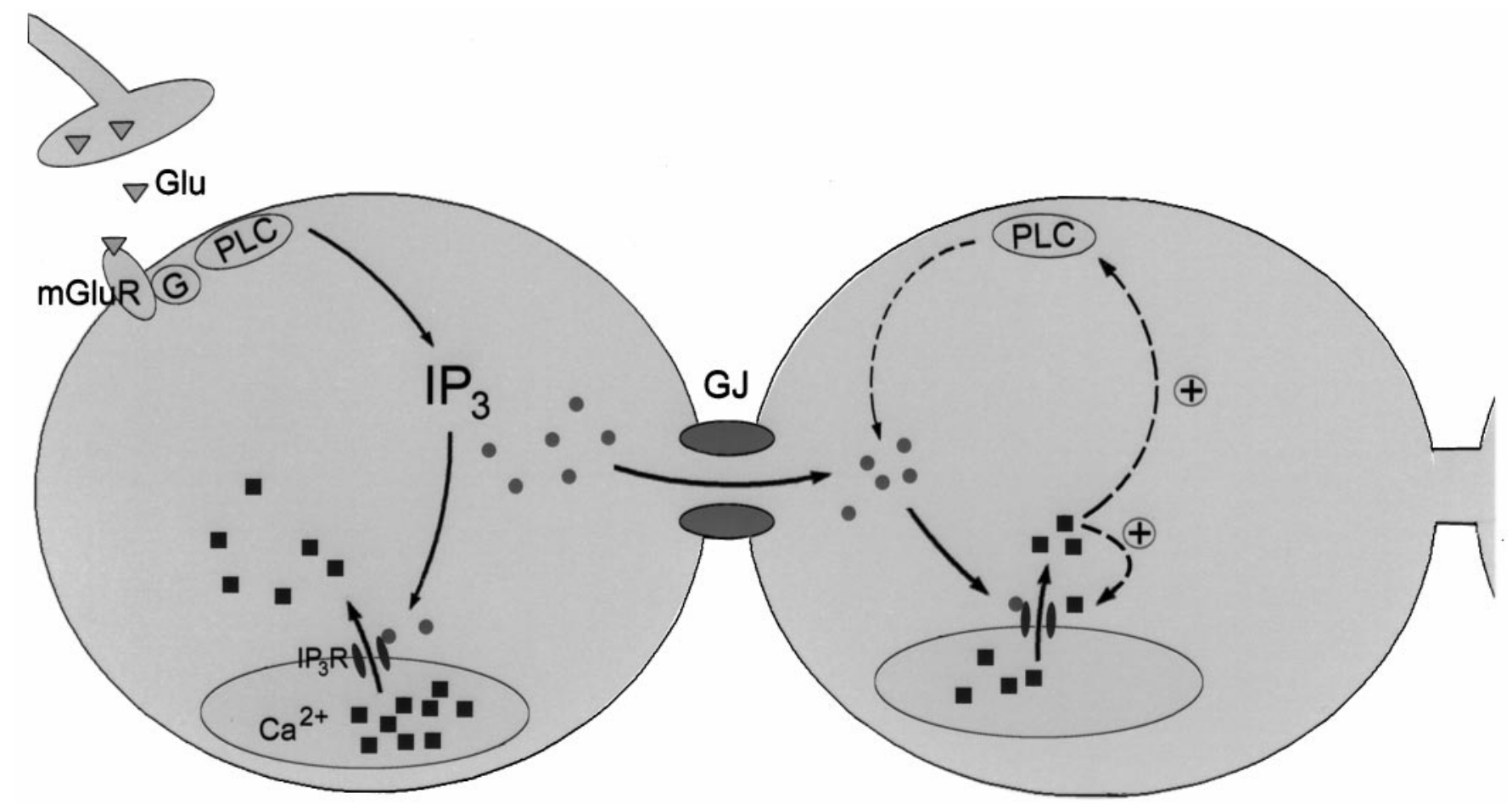

Figure 6. Model for the initiation and propagation of interneuronal calcium waves underlying neuronal domains. Waves are initiated either by ambient or synaptically released glutamate (Glu; open triangles) that acts on metabotropic glutamate receptors $(m G l u R)$. This stimulates the G-protein $(G)$-phospholipase $\mathrm{C}(P L C)$ cascade that results in the production of inositol trisphosphate $\left(I P_{3} ;\right.$ filled circles $) . I P_{3}$ activates $I P_{3}$ receptors $\left(I P_{3} R\right.$; small filled ovals) and thereby causes calcium release ( filled squares) from intracellular stores. In addition, $I P_{3}$ also diff uses through gap junctions $(G J)$ into neighboring neurons where it causes calcium release. In coupled cells, $I P_{3}$ could be regenerated by calcium-mediated positive feedback loops $($ dashed arrows), including the sensitization of $I P_{3} R$ and stimulation of $P L C$.

calcium response, $\mathrm{IP}_{3}$, or one of its metabolites (Jia et al., 1995; for review, see Berridge, 1993), most likely also acts as the intercellular messenger that is responsible for intercellular wave propagation. The propagation of calcium waves over many cells, without significant decrement (Fig. 5A; Yuste et al., 1995), implies the involvement of regenerative mechanisms. Although the specific nature of these mechanisms remains to be determined for neuronal domains, calcium-induced increases in $\mathrm{IP}_{3}$ receptor sensitivity (Bezprozvanny et al., 1991) and in phospholipase C activity (Meyer, 1991) could provide the necessary positive feedback loops.

The cellular mechanisms of intercellular neuronal calcium waves in the developing neocortex closely resemble mechanisms that have been proposed for calcium waves in non-neuronal systems (for review, see Sanderson, 1995). In this respect, young neurons, still at a stage when they are sparsely connected by synapses (Miller, 1988), use mechanisms typical of nonelectrically excitable cells to establish cellular communication and coordinate behavior. Currently, there are only a few studies that address the cellular mechanisms of gap junction-mediated intercellular calcium waves between mammalian neurons, despite the presence of extensive neuronal coupling early in development. Recently Charles et al. (1996) proposed a model for intercellular calcium waves between cultured cortical neurons and GT1-1immortalized neurons. In contrast to our model, their model proposes that neuronal calcium waves propagate by the intercellular spread of membrane depolarizations and the subsequent activation of voltage-gated $\mathrm{Ca}^{2+}$ channels and influx of extracellular calcium. However, despite some similarities between calcium waves in slices and calcium waves in cultured cells, such as propagation speed [ $\sim 100 \mu \mathrm{m} / \mathrm{sec}$ in slices (Yuste et al., 1995) and 100-200 $\mu \mathrm{m} / \mathrm{sec}$ in cultures (Charles et al., 1996)] or area of propagation ( $\sim 3000 \mu \mathrm{m}^{2}$ in slices and $50-100$ cells in culture), there also exist several basic differences between these waves. Waves in cortical slices are initiated by mGluRs, whereas those in cultures require mechanical stimulation. Moreover, waves in slices are TTX-insensitive, whereas those in cultures are TTXsensitive. These fundamental differences indicate that gap junctions between cortical neurons can support different types of calcium waves depending on the environmental conditions. Because coupling strength and type of expressed connexins are regulated by factors such as neuromodulators (for review, see Bennett et al., 1991) or injury (Gutnick et al., 1985; Rohlmann et al., 1994; Balice-Gordon et al., 1996), the coupling situation in cultured cortical cells might be quite different than that in acute brain slices.

\section{Direct intercellular diffusion of second messengers: an alternative route of neuronal communication}

Since the discovery of electrical synapses by Furshpan and Potter (1959) almost 40 years ago, neuronal gap junctions have primarily been viewed as the basis of electrical synapses dedicated to electronically coupling cells. However, electrical coupling between developing neurons is generally very weak, raising doubts about their contribution to neuronal synchronization (for review, see Katz, 1995; Kandler, 1997). In contrast to the small changes in the electrical potential that such coupling can elicit, we found that regenerative biochemical waves, spreading over considerable distances, can be supported by such apparently weak coupling. The direct exchange of $\mathrm{IP}_{3}$ between neurons provides another avenue, 
besides the conventional role of electrical activity, by which neuronal behavior can be synchronized among members of neuronal assemblies. Although our data do not exclude the possibility that weak electrical communication between cortical neurons (Connors et al., 1983; Lo Turco and Kriegstein, 1991; Peinado et al., 1993b) coexists with biochemical communication, biochemical communication seems to be the main functional route by which coupled cortical neurons synchronize their behavior. Because neuronal coupling is most prominent before and during the major period of synapse formation (Connors et al., 1983; Lo Turco and Kriegstein, 1991; Peinado et al., 1993a; Kim et al., 1995) and is inversely correlated with synaptic activity in ferrets (Kandler and Katz, 1998), coordination of biochemical activity across large cell assemblies is likely to influence cortical development before or during early stages of synapse formation but not after the emergence of bona fide circuits. Because of the widespread effects of second messengers on essential cellular processes such as neuronal differentiation and gene expression (Spitzer, 1995; Finkbeiner and Greenberg, 1996), the direct control of biochemical activity can coordinate a much wider range of cellular functions than would be possible by the coordination of electrical activity alone.

\section{REFERENCES}

Abe T, Sugihara H, Nawa H, Shigemoto R, Mizuno N, Nakanishi S (1992) Molecular characterization of a novel metabotropic glutamate receptor mGluR5 coupled to inositol phosphate/Ca ${ }^{2+}$ signal transduction. J Biol Chem 267:13361-13368.

Allbritton NL, Meyer T (1993) Localized calcium spikes and propagating calcium waves. Cell Calcium 14:691-697.

Balice-Gordon RJ, Pereda A, Pinter MJ (1996) Functional gap junctions couple motor neurons in development and reinnervation. Soc Neurosci Abstr 22:1487.

Baux G, Simonneau M, Tauc L, Segundo JP (1978) Uncoupling of electrotonic synapses by calcium. Proc Natl Acad Sci USA 75:4577-4581.

Bennett MV, Goodenough DA (1978) Gap junctions, electrotonic coupling, and intercellular communication. Neurosci Res Program Bull 16:1-486.

Bennett MV, Barrio LC, Bargiello TA, Spray DC, Hertzberg E, Saez JC (1991) Gap junctions: new tools, new answers, new questions. Neuron 6:305-320.

Berridge MJ (1993) Inositol trisphosphate and calcium signaling. Nature 361:315-325.

Bevilacqua JA, Downes CP, Lowenstein PR (1995) Transiently selective activation of phosphoinositide turnover in layer $\mathrm{V}$ pyramidal neurons after specific mGluRs stimulation in rat somatosensory cortex during early postnatal development. J Neurosci 15:7916-7928.

Bezprozvanny I, Watras J, Ehrlich BE (1991) Bell-shaped calciumresponse curves of Ins(1,4,5)P3- and calcium-gated channels from endoplasmic reticulum of cerebellum. Nature 351:751-754.

Blanton MG, Lo Turco JJ, Kriegstein AR (1989) Whole cell recording from neurons in slices of reptilian and mammalian cerebral cortex. J Neurosci Methods 30:203-210.

Boitano S, Dirksen ER, Sanderson MJ (1992) Intercellular propagation of calcium waves mediated by inositol trisphosphate. Science 258:292-295.

Catania MV, Landwehrmeyer GB, Testa CM, Standaert DG, Penney Jr JB, Young AB (1994) Metabotropic glutamate receptors are differentially regulated during development. Neuroscience 61:481-495.

Challiss RA, Mistry R, Gray DW, Nahorski SR (1994) Modulatory effects of NMDA on phosphoinositide responses evoked by the metabotropic glutamate receptor agonist $1 \mathrm{~S}, 3 \mathrm{R}-\mathrm{ACPD}$ in neonatal rat cerebral cortex. Br J Pharmacol 112:231-239.

Chapman B, Stryker MP, Bonhoeffer T (1996) Development of orientation preference maps in ferret primary visual cortex. J Neurosci 16:6443-6453.

Charles AC, Kodali SK, Tyndale RF (1996) Intercellular calcium waves in neurons. Mol Cell Neurosci 7:337-353.

Christ GJ, Moreno AP, Melman A, Spray DC (1992) Gap junctionmediated intercellular diffusion of $\mathrm{Ca}^{2+}$ in cultured human corporal smooth muscle cells. Am J Physiol 263:C373-C383.
Connors BW, Benardo LS, Prince DA (1983) Coupling between neurons of the developing rat neocortex. J Neurosci 3:773-782.

Dudek SM, Bowen WD, Bear MF (1989) Postnatal changes in glutamate stimulated phosphoinositide turnover in rat neocortical synaptoneurosomes. Brain Res 47:123-128.

Feller MB, Wellis DP, Stellwagen D, Werblin FS, Shatz CJ (1996) Requirement for cholinergic synaptic transmission in the propagation of spontaneous retinal waves. Science 272:1182-1187.

Finkbeiner S, Greenberg ME (1996) $\mathrm{Ca}^{2+}$-dependent routes to Ras: mechanisms for neuronal survival, differentiation, and plasticity. Neuron 16:233-236.

Fotuhi M, Sharp AH, Glatt CE, Hwang PM, von Krosigk M, Snyder SH, Dawson TM (1993) Differential localization of phosphoinositidelinked metabotropic glutamate receptor (mGluR1) and the inositol 1,4,5-trisphosphate receptor in rat brain. J Neurosci 13:2001-2012.

Furshpan EJ, Potter DD (1959) Transmission at the giant motor synapses of the crayfish. J Physiol (Lond) 145:289-325.

Galli L, Maffei L (1988) Spontaneous impulse activity of rat retinal ganglion cells in prenatal life. Science 242:90-91.

Giffin K, Solomon JS, Burkhalter A, Nerbonne JM (1991) Differential expression of voltage-gated calcium channels in identified visual cortical neurons. Neuron 6:321-332.

Gu X, Olson EC, Spitzer NC (1994) Spontaneous neuronal calcium spikes and waves during early differentiation. J Neurosci 14:6325-6335.

Gutnick MJ, Lobel-Yaakov R, Rimon G (1985) Incidence of neuronal dye-coupling in neocortical slices depends on the plane of section. Neuroscience 15:659-666.

Horton JC, Hocking DR (1996) An adult-like pattern of ocular dominance columns in striate cortex of newborn monkeys prior to visual experience. J Neurosci 16:1791-1807.

Jia WW, Liu Y, Cynader M (1995) Postnatal development of inositol 1,4,5-trisphosphate receptors: a disparity with protein kinase C. Brain Res 85:109-118.

Kandler K (1997) Coordination of neuronal activity by gap junctions in the developing neocortex. Semin Dev Biol 8:43-51.

Kandler K, Katz LC (1995) Mechanisms for initiation and propagation of intercellular calcium waves in the developing visual cortex. Soc Neurosci Abstr 21:1285.

Kandler K, Katz LC (1998) Relationship between dye coupling and spontaneous activity in developing ferret visual cortex. Dev Neurosci, in press.

Kano M, Garaschuk O, Verkhratsky A, Konnerth A (1995) Ryanodine receptor-mediated intracellular calcium release in rat cerebellar Purkinje neurones. J Physiol (Lond) 487:1-16.

Kasai H, Peterson OH (1994) Spatial dynamics of second messengers: IP3 and cAMP as long range and associative messengers. Trends Neurosci 17:95-101.

Katz LC (1995) Coordination of vertebrate cellular assemblies by gap junctions. Semin Dev Biol 6:117-125.

Katz LC, Shatz CJ (1996) Synaptic activity and the construction of cortical circuits. Science 274:1133-1138.

Keirstead SA, Miller RF (1995) Calcium waves in dissociated retinal glial (Muller) cells are evoked by release of calcium from intracellular stores. Glia 14:14-22.

Kendall DA, Nahorski SR (1987) Acute and chronic lithium treatments influence agonist and depolarization-stimulated inositol phospholipid hydrolysis in rat cerebral cortex. J Pharmacol Exp Ther 241:1023-1027.

Kim HG, Fox K, Connors BW (1995) Properties of excitatory synaptic events in neurons of primary somatosensory cortex of neonatal rats. Cereb Cortex 5:148-157.

Lo Turco JJ, Kriegstein AR (1991) Clusters of coupled neuroblasts in embryonic neocortex. Science 252:563-566.

Lo Turco JJ, Blanton MG, Kriegstein AR (1991) Initial expression and endogenous activation of NMDA channels in early neocortical development. J Neurosci 11:792-799.

Meister M, Wong ROL, Baylor DA, Shatz CJ (1991) Synchronous bursts of action potentials in ganglion cells of the developing mammalian retina. Science 252:939-943.

Meyer T (1991) Cell signaling by second messenger waves. Cell 64:675-678.

Miller MW (1988) Development of projection and local circuit neurons in neocortex. In: Development and maturation of cerebral cortex (Peters A, Jones EG, eds), pp 133-175. New York: Plenum.

Peinado A, Yuste R, Katz LC (1993a) Extensive dye coupling between 
rat neocortical neurons during the period of circuit formation. Neuron 10:103-114.

Peinado A, Yuste R, Katz LC (1993b) Gap junctional communication and the development of local circuits in neocortex. Cereb Cortex 3:488-498.

Peracchia C (1978) Calcium effects on gap junction structure and cell coupling. Nature 271:669-671.

Rao G, Barnes CA, McNaughton BL (1987) Occlusion of hippocampal electrical junctions by intracellular calcium injection. Brain Res 408:267-270.

Rohlmann A, Laskawi R, Hofer A, Dermietzel R, Wolff JR (1994) Astrocytes as rapid sensors of peripheral axotomy in the facial nucleus of rats. NeuroReport 5:409-412.

Saez JC, Connor JA, Spray DC, Bennett MVL (1989) Hepatocyte gap junctions are permeable to the second messenger, inositol 1,4,5trisphosphate, and to calcium ions. Proc Natl Acad Sci USA $86: 2708-2712$.

Sanderson MJ (1995) Intercellular calcium waves mediated by inositol trisphosphate. Ciba Found Symp 188:175-189.

Shatz CJ (1983) The prenatal development of the cat's retinogeniculate pathway. J Neurosci 3:482-499.
Shatz CJ, Stryker MP (1988) Prenatal tetrodotoxin infusion blocks segregation of retinogeniculate afferents. Science 242:87-89.

Simpson PB, Challiss RA, Nahorski SR (1995) Neuronal $\mathrm{Ca}^{2+}$ stores: activation and function. Trends Neurosci 18:299-306.

Spitzer NC (1995) Spontaneous activity: functions of calcium transients in neuronal differentiation. Perspect Dev Neurobiol 2:379-386.

Veenstra RD (1996) Size and selectivity of gap junction channels formed from different connexins. J Bioenerg Biomembr 28:327-337.

Warner A (1992) Gap junctions in development-a perspective. Semin Cell Biol 3:81-91.

Weliky M, Katz LC (1997) Disruption of orientation tuning in visual cortex by artificially correlated neuronal activity. Nature 386:680-685.

Wolszon LR, Rehder V, Kater SB, Macagno ER (1994) Calcium wave fronts that cross gap junctions may signal neuronal death during development. J Neurosci 14:3437-3448.

Yuste R, Peinado A, Katz LC (1992) Neuronal domains in developing neocortex. Science 257:665-669.

Yuste R, Nelson DA, Rubin WW, Katz LC (1995) Neuronal domains in developing neocortex: mechanisms of coactivation. Neuron 14:7-17. 